Sharif University of Technology
Scientia Iranica
SCIENTIA
I RAN I CA
Transactions A: Civil Engineering
www.scientiairanica.com

\title{
Probabilistic seismic hazard analysis using reliability methods
}

\author{
M. Kia and M. Banazadeh* \\ Department of Civil and Environmental Engineering, Amirkabir University of Technology, Tehran, P.O. Box 15875-4413, Iran.
}

Received 2 July 2015; received in revised form 12 January 2016; accepted 26 April 2016

\section{KEYWORDS \\ Probabilistic seismic hazard analysis; \\ Reliability methods; \\ Probabilistic model; \\ Epistemic and \\ aleatory uncertainties; \\ Limit-state function.}

\begin{abstract}
By considering uncertainties in the input parameters (e.g., magnitude, location, wave path, etc.), the Probabilistic Seismic Hazard Analysis (PSHA) aims to compute annual rate of various exceeding ground motions at a site or a map of sites of all anticipated given earthquakes. Uncertainties may be originated due to inherent randomness of the phenomena or variability in the mean values of different models parameters, mainly due to use of finite-sample size of observations. The first, in literature reviews, is commonly named aleatory uncertainty; the second is known as epistemic uncertainty. The total probability numerical integration, generally employed to calculate PSHA, only considers aleatory uncertainties, and variability in the models' parameters is neglected to simplify calculation. In this paper, as an alternative to the total probability numerical integration, matured and standard reliability methods tailored to effortlessly consider both types of uncertainties are put forward to compute site-specific PSHA. Then, as an application study, the peak ground acceleration hazard curve for the site, at which a historical bridge is located, is developed and compared with those obtained from the total probability numerical integration.
\end{abstract}

(C) 2017 Sharif University of Technology. All rights reserved.

\section{Introduction}

The Probabilistic Seismic Hazard Analysis (PSHA) is an approach broadly applied to describe probable future ground shakings at a site. In this approach, annual rate of exceeding for a given ground motion hazard parameter $\lambda(\mathrm{im})$ at a specific site is estimated by quantifying uncertainties in the characteristics of seismic source such as magnitude, distance, and wave pathway. The mathematical description of the PSHA was first set up by Cornell [1]. The formulation was based on total probability theorem. Eq. (1) demonstrates general form of the formulation:

*. Corresponding author. Tel./Fax: +982164543049 E-mail addresses: mehdikia@aut.ac.ir (M. Kia); mbanazadeh@aut.ac.ir (M. Banazadeh)

$$
\begin{aligned}
& \lambda(i m)= \sum_{i=1}^{n} v_{i} P(I M>i m) \\
&= \sum_{i=1}^{n} v_{i} \iint P(I M>i m \mid M=m \\
&R=r) f_{M}(m) \times f_{R}(r) d m . d r
\end{aligned}
$$

where $v_{i}$ is the mean annual rate of occurrence of all earthquakes which are greater than or equal to the minimum magnitude, $I M$ denotes a ground motion hazard parameter. Since, in this study, there is more emphasis on the methodology rather than the hazard parameter, the well-known Peak Ground Acceleration $(P G A)$ is chosen as the ground motion measure. $n$ describes number of seismic sources and $P(I M>i m \mid M=m, R=r)$ is the conditional 
probability of exceeding the chosen $I M$ for a given magnitude and distance. In this term, dispersion around the mean value of the $I M$ is addressed using lognormal distribution. Finally, $f_{M}(m)$ and $f_{R}(r)$ indicate probability density functions of magnitude and distance, respectively. These probabilistic models reflect variability inherent in the characteristics of seismic source (i.e., magnitude and location). However, probabilistic models developed based on finite-sample size observations pose another type of uncertainty, which is known as epistemic uncertainty. This type of uncertainty is technically considered by employing probabilistic model with random, rather than constant, parameters. It is obvious that considering both mentioned uncertainties in the conventional framework makes calculation extremely difficult. Since impacts of epistemic uncertainties on performance evaluation and design have recently received serious attention [2], employing a framework, which is capable of considering both types of uncertainties, would be interesting.

Recently, reliability framework has been widely applied in literature to explicitly address aleatory and epistemic uncertainties in engineering reliability analysis and reliability-based design [3-6]. Haukaas [7] proposed the "unified reliability analysis" as an alternative to the seismic risk analysis based on conditional probabilities. In this approach, First Order Reliability Method (FORM) is implemented to carry out design optimization for a system with limit-state function developed in terms of monetary loss. Du [8] quantified the effects of the aleatory and epistemic uncertainties with belief and plausibility measures in the context of the evidence theory. $\mathrm{He}$ implemented the first order reliability method for probabilistic analysis and nonlinear optimization for interval analysis to handle black-box functions. As an application of the methodology proposed by Haukaas [7], Bohl [9] developed loss curve for an example building and compared the result with the one obtained from Pacific Earthquake Engineering Research (PEER) center approach. Der Kiureghian and Ditlevsen [10] focused on the influence of the two types of uncertainties on reliability assessment, codified design, performance-based engineering, and risk-based decision-making. Two examples were studied to demonstrate the influence of statistical dependence arising from epistemic uncertainties on systems and time-variant reliability problems. Koduru and Haukaas [11] studied the viability of the FORM in the context of both static and dynamic finite-element problems. It was concluded that FORM is not an appropriate reliability method for time-variant reliability problems. Xiao et al. [12] proposed an optimization model for sensitivity analysis under both aleatory and epistemic uncertainties. The parameters with sufficient information were modeled using probability distributions, while others were modeled using a pair of upper and lower cumulative distributions (the so-called P-box). Mahsuli and Haukaas [13] developed an open-source reliability software, named "Rt" , as a computational platform for evaluating reliability problems. This software, which is available on the following website link "http://www.inrisk.ubc.ca", is employed in the present study to solve reliability-based PSHA problem.

According to the above description, in this paper, reliability framework as an alternative to the conventional approach is put forward to address problems in Eq. (1). Contrary to the conventional approach, this framework is able to effortlessly and explicitly address both aleatory and epistemic uncertainties. Magnitude model with random parameters is defined as an example to address the ability of the framework to reflect both types of uncertainties within calculation of PSHA. This ability would be more notable when attenuation relations with random model parameters are implemented in calculating PSHA. Furthermore, availability of reliability software, such as "RT", effortlessly capable of carrying-out reliability analysis, makes the reliability-based PSHA more interesting and appealing. This paper starts with describing reliability analysis and limit-state function in detail. Next, magnitude and location models are developed to be applied to conventional and reliability-based framework to develop seismic hazard curve. The results are presented and compared at the end to provide insight into the accuracy and efficiency of the suggested reliability methods.

\section{Reliability analysis}

In classical structural reliability analysis, the system reliability $R$ and probability of failure $p_{f}$ are defined as $[4-6]$ :

$$
\begin{aligned}
& R=P[G(X) \geq 0]=\int_{g(X) \geq 0} \ldots \int f_{X}(X) d X, \\
& p_{f}=P[G(X)<0]=\int_{g(X)<0} \ldots \int f_{X}(X) d X,
\end{aligned}
$$

where $P[$.$] denotes a probability indicator, G($.$) is$ a limit-state function which defines the performance event for which the probability is being assessed, and $X=\left(X_{1}, X_{2}, \ldots, X_{n}\right)$ is the vector of random variables. $f_{X}(X)$ denotes the joint probability density function (pdf) of $X$. Reliability is graphically interpreted as the volume underneath the surface (hyper-surface for higher than 2-D problems) of $f_{X}(X)$ on the side of safe region [6] (Figure 1). The direct evaluation of the probability integration is extremely difficult. The main reasons given in different reliability texts are [3-5]: 


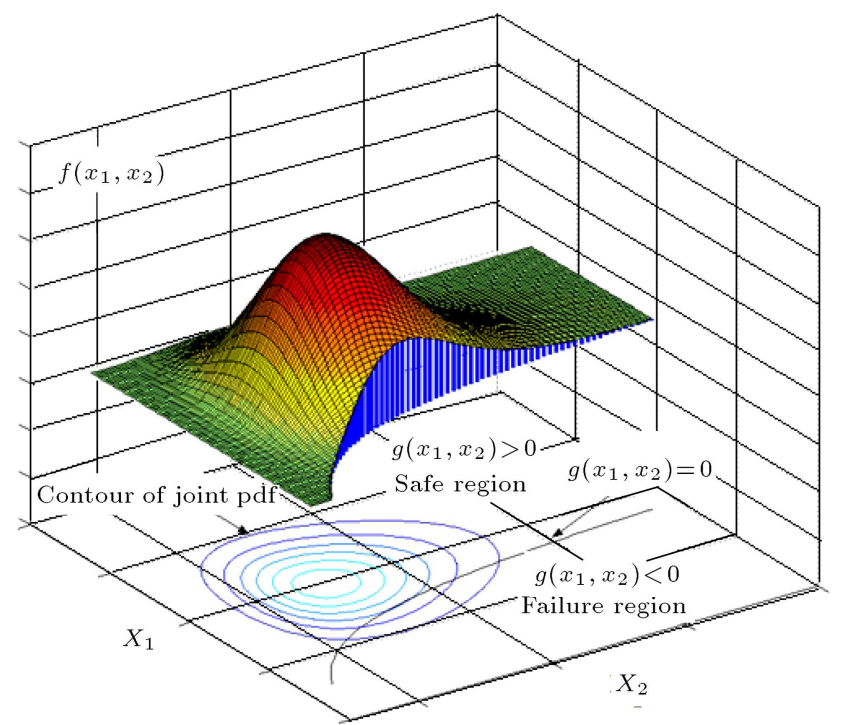

Figure 1. Graphical interpretation of the probability integration [6].

- $f_{X}(X)$ is practically impossible to obtain; evaluating the multiple integral is difficult even if this information is available.

- In many engineering applications, integration boundary (i.e., $G(X)=0$ ) is not defined explicitly. Even if this function is available, it is usually a nonlinear and complicated function of $X$.

According to the above reasons, numerical solutions are the only choice available. Several reliability methods are introduced to estimate the probability integration numerically. Gradient-based methods, i.e. FORM and Second-Order Reliability Method (SORM) along with more sophisticated Monte Carlo Simulation (MCS), are such techniques broadly applied to solve the probability integration in Eq. (3). MCS generates random outcomes of the random Variables and evaluates $G(X)$ for each realization. Then, it counts the number of times a failure occurred and estimates the probability of failure by $[3,5,9]$ :

$$
p_{f}=\frac{\text { Number of failure }}{\text { Total number of sampling }} .
$$

This method is extremely robust. It can solve probability integration in Eq. (3) with any type of limit-state functions, even those with discontinuities. However, the main disadvantage of MCS is that it requires a large number of evaluations of the limit-state function to obtain the failure probability. Hence, the MCS is time-consuming, especially for random event with small probability of failure. This deficiency reduces the use of MCS in structural reliability analysis. In contrast, gradient-based methods are highly efficient due to their search for only the point with the highest contribution to the probability integration; no time is

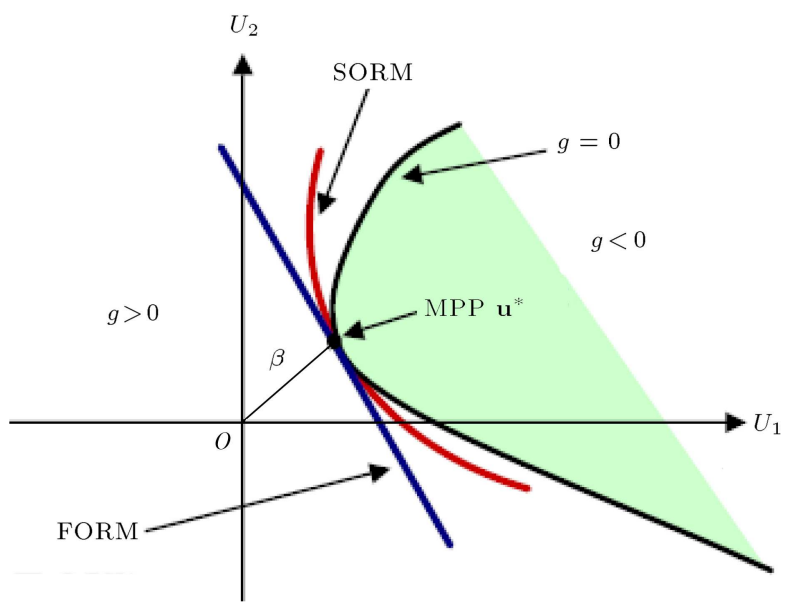

Figure 2. Comparison of the first order and second reliability methods [6].

wasted in calculations and they do not significantly contribute to the probability of failure. This characteristic of the gradient-based methods making them tailored to compute probability of rare event would be greatly interesting for practical purposes. Since the approximation of the limit-state function in SORM is better than that in FORM (Figure 2), SORM is generally more accurate than FORM, especially for highly nonlinear limit-state function. However, SORM is not as efficient as FORM due to computing the second-order derivatives. Therefore, a great number of solutions are proposed to improve efficiency of the SORM using various quadratic approximations, one of which is the Breitung's improved formula using the theory of asymptotic approximations [14]. This simple closed-form solution, applied to the present study as the second-order analysis, modifies failure probability of the FORM using the first principle curvature. Generally, gradient-based methods take the three following steps to estimate probability of failure $[3,6,12]$.

1. The vector of random variables, $X=\left(X_{1}, X_{2}, \ldots\right.$, $\left.X_{n}\right)$, in their original random space is transferred into independent standard normal vector, $U=\left(U_{1}, U_{2}, \ldots, U_{n}\right)$, in standard normal space $(U$ space). After transformation, limit-state function $G(X)$ is changed to $\tilde{G}(U)$ in $U$-space. Note that mathematical formulation of limit-state function after transformation is different from that in the original space.

2. Next, a point located on $\tilde{G}(U)=0$ and has the highest contribution to the probability integration must be sought. In reliability text, this point is named Maximum Probable Point (MPP), or design point, and computed by an iterative optimization process as: 


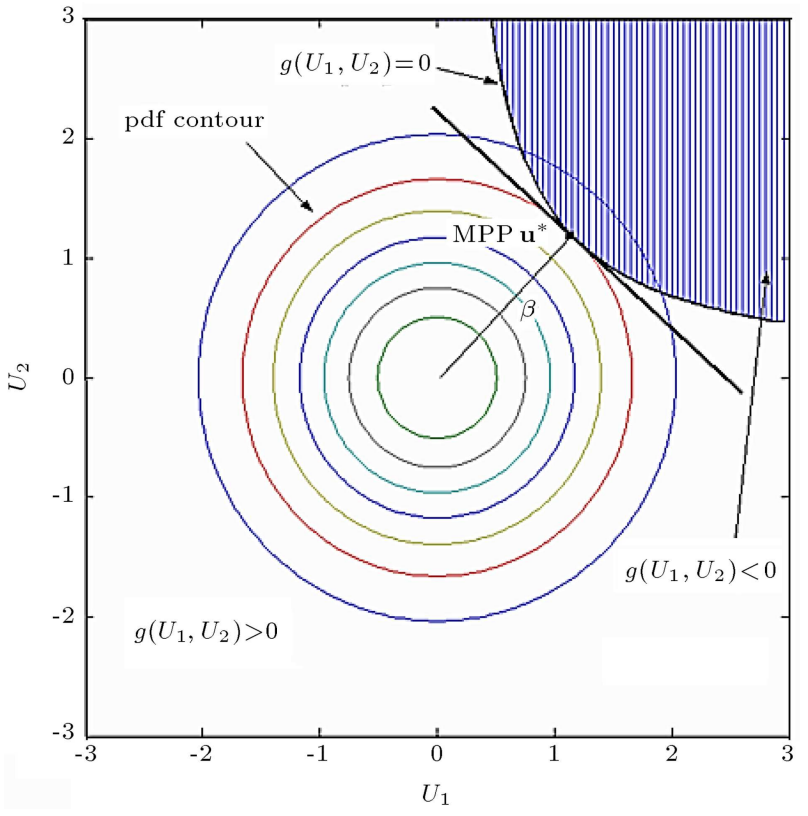

Figure 3. Probability integration in the $U$-space [6].

$$
\left\{\begin{array}{l}
\operatorname{Max} \tilde{f}(U)=\Pi_{i=1}^{n} \frac{1}{\sqrt{2 \pi}} \operatorname{Exp}\left(-\frac{u_{i}^{2}}{2}\right) \\
\text { Subjected to } \tilde{G}_{L}(U)=0
\end{array}\right.
$$

where $\tilde{f}(U)$ demonstrates the mathematical description of the integrand in $U$-space. Since $\Pi_{i=1}^{n} \frac{1}{\sqrt{2 \pi}} \operatorname{Exp}\left(-\frac{u_{i}^{2}}{2}\right)=\frac{1}{\sqrt{2 \pi}} \operatorname{Exp}\left(-\frac{\sum_{i=1}^{n} u_{i}^{2}}{2}\right), \max -$ imizing $\Pi_{i=1}^{n} \frac{1}{\sqrt{2 \pi}} \operatorname{Exp}\left(-\frac{u_{i}^{2}}{2}\right)$ is equivalent to minimizing $\sum_{i=1}^{n} u_{i}^{2}$. Accordingly, MPP is the point on the integral boundary, i.e. $\tilde{G}(U)=0$, which has the shortest distance to the origin in $U$-space (Figure 3). In technical texts, this minimum distance is called reliability index and is shown by $\beta$.

3. Finally, based on the obtained reliability index, the probability of failure is estimated by the following equations:

$$
p_{f}=\left\{\begin{array}{c}
\Phi(-\beta) \rightarrow \text { If FORM selected } \\
\Phi(-\beta) \Pi_{i=1}^{n-1}\left(1+\beta \kappa_{i}\right)^{\frac{1}{2}} \rightarrow \text { If SORM } \\
\quad \text { Breitung's improved formulation }) \\
\text { selected }
\end{array}\right.
$$

where $\kappa_{i}$ denotes the principal curvatures of the limit-state function, i.e. $\tilde{G}(U)$, at the minimum distance point.

\section{Limit-state function}

According to the previous section, limit-state function defining desired event is the core of the reliability methods. A limit-state function consists of two main parts: threshold and probabilistic models. Threshold is the value assigned by analyst, but probabilistic model is an equation (or algorithm) that simulates all possible scenarios of physical phenomena. In addition, probabilistic models implemented in a reliability analysis should have other characteristics that are fully described in $[7,9,13]$. A few of these characteristics include: accounting for both aleatory and epistemic uncertainties in terms of random variables and being continuously differentiable. Since the foundation of all reliability algorithms are based on several trial realizations of random variables, probabilistic models must be able to yield deterministic output(s) for a given realization of input variables. The exact number of realizations depends on the efficiency of the algorithm employed to solve a reliability problem.

Depending on the objective performance, the number of probabilistic models required to define desired limit-state function would be different. For example, in a seismic risk analysis which computes exceedance probability of monetary loss, three intensities, demand, and consequence models are required to develop limit-state function. But, for seismic hazard analysis which is the subject of this paper, only probabilistic intensity model would be adequate. Therefore, following limit-state function is extended in this paper to address the problem of calculating Eq. (1):

$$
G()=p g a-P G A(\Theta, M, R),
$$

where pga indicates peak ground acceleration threshold, $\operatorname{pga}(\Theta, M, R)$ represents an intensity model predicting peak ground acceleration at a designated site. In this model, $\Theta, M$, and $R$ indicate model parameters, earthquake magnitude, and wave propagation effects, respectively. Fortunately, most of attenuation relations proposed in the literature comply with the mentioned characteristics of a probabilistic model and can be directly amenable to a reliability analysis. One example is the following ground-shaking intensity model predicting peak ground acceleration at a site [15] and implemented in this paper to describe reliabilitybased PSHA. It is noteworthy that analyst would be autonomous in the use of his developed or other probabilistic intensity model:

$$
\begin{aligned}
\log (P G A)= & a M_{w}-b \sqrt{\left(R^{2}+H^{2}\right)} \\
& -d \cdot \log \left(\sqrt{R^{2}+H^{2}}\right)+c_{i} s_{i}+\sigma . \varepsilon .
\end{aligned}
$$

In the above equation, $a, b, c_{i}$, and $s_{i}$ are the parameters of the intensity model; $M_{w}, R$, and $H$ represent earthquake magnitude, focal depth, and epicentral distance, respectively; $\varepsilon$ is the standard normal random variable representing the model error; and $\sigma$ is the standard deviation of the model error. Furthermore, the model yields a unique deterministic value of $P G A$ for a given 
realization of input variables and is continuously differentiable. Hence, Eq. (8) can be directly implemented in reliability analysis. It is worthwhile to mention that the above model is not capable of addressing epistemic uncertainty due to use of constant parameters. In other words, variability in the model parameters, i.e. $a, b, c_{i}$, and $s_{i}$, is ignored when the model is developed. By utilizing the above relationship, the limit-state function $G()$ is rewritten to address the problem of calculating the probability that $P G A$ at a site exceeds a specific threshold value:

$$
\begin{aligned}
& G()= \\
& \left.p g a-10\left[a M_{w}-b \sqrt{\left(R^{2}+H^{2}\right)}-d \cdot \log \sqrt{\left(R^{2}+H^{2}\right.}\right)+c_{i} s_{i}+\sigma \cdot \varepsilon\right] .
\end{aligned}
$$

\section{Case study example}

In this section, both traditional and reliability-based methodologies are implemented to develop peak ground acceleration hazard curve at a site of a historical bridge. This bridge, dating back to about 250 years, is located at $52.665^{\circ}$ east and $36.525^{\circ}$ north. Seismicity catalog of the site was first developed based on all historical and systematic earthquakes occurred at a distance up to $150 \mathrm{~km}$ from the site. For the sake of simplicity, three circular area sources with different radii were defined. Only major excitations were considered, and near-source effects are limited by defining lower bound radius being equal to $10 \mathrm{~km}$ (Figure 4). Next, both magnitude and location probability functions are developed for each seismic zone. These probability density functions are then utilized in the intensity model proposed in the previous section to formulate limit-state function, Eq. (9), for each seismic zone. After extending limit-state function, reliability methods are employed to calculate the probability that peak ground acceleration exceeds certain ground motion levels.

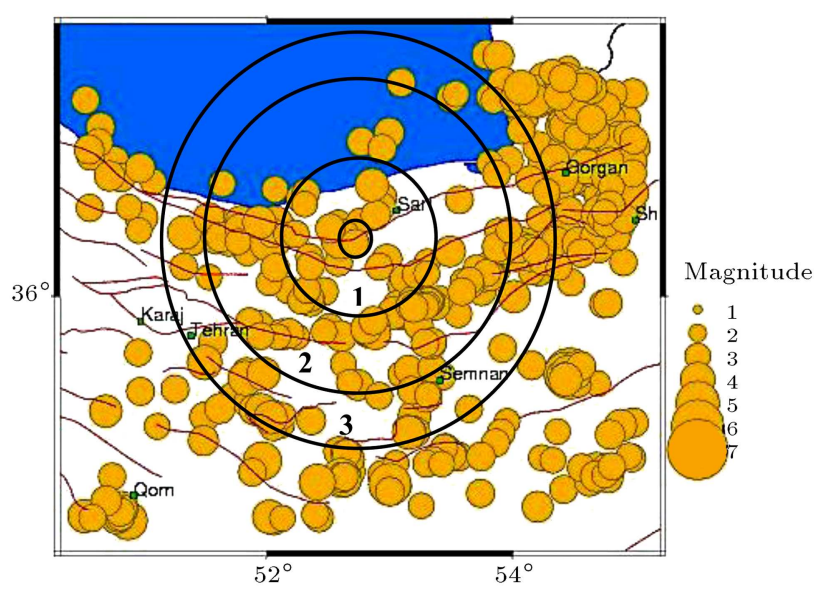

Figure 4. Concentric circular seismic sources.

\subsection{Magnitude model}

The relative frequency of various-sized earthquakes has been usually specified by a truncated exponential distribution function developed based on Gutenberg and Richter's recurrence law:

$$
\operatorname{Ln}(N(m))=a-b \cdot m \rightarrow N(m)=e^{a} \cdot e^{-b \cdot m},
$$

where $N(m)$ indicates the number of earthquake with magnitude equal to or greater than " $m$ "; " $a$ " and " $b$ " are the recurrence model parameters. According to Eq. (10), cumulative distribution function of earthquake magnitude and consequently its probability distribution function can be computed as:

$$
\begin{aligned}
F_{m}(m) & =P\left(M \leq m \mid m_{\min }<M<m_{\max }\right) \\
& =\frac{\left(n\left(m_{\min }\right)-n(m)\right)}{n\left(m_{\min }\right)-n\left(m_{\max }\right)} \\
& =\frac{1-e^{-b\left(m-m_{\min }\right)}}{1-e^{-b\left(m_{\max }-m_{\min }\right)}}, \\
f_{m}(m) & =\frac{\partial F_{m}(m)}{\partial m}=\frac{b \cdot e^{-b\left(m-m_{\min }\right)}}{1-e^{-b\left(m_{\max }-m_{\min }\right)}} \\
m_{\min } & <m<m_{\max }
\end{aligned}
$$

where $m_{\text {min }}$ indicates lower bound value of earthquake magnitude selected based on engineering judgment and seismicity catalog of the site; $m_{\max }$ is the upper bound value of magnitude used to demonstrate that maximum magnitude may be generated by seismic source. Magnitude model parameters, i.e. $m_{\max }, b$, and $m_{\text {min }}$, for each of the above three seismic zones are presented in Table 1. Except for the latter, the other parameters are computed by a sophisticated procedure which considers uncertainty in the input magnitude data $[16,17]$.

\subsection{Location model}

To predict ground motion intensity at a site location, it is also necessary to describe probability distribution of site to source distance as a more common parameter applied to reflect wave propagation effects. Since rupture can be extended over ten kilometers, various distance measures have been developed to estimate ground motion parameters at a site (Figure 5). In Eq. (9), the epicentral distance $R$ associated with focal depth $H$ is used to reflect wave propagation effects. Probabilistic description of epicentral distance can be easily computed by assuming that earthquakes occur with equal probability anywhere at a seismic zone. Considering the above assumption, the probability of locating an epicenter at a distance of less than " $r$ " and greater than $r_{\min }$ can be calculated by: 
Table 1. Parameters of magnitude model for each seismic zone.

\begin{tabular}{ccccc}
\hline Seismic-zone & Model parameter & Median & Standard deviation & $\begin{array}{c}\boldsymbol{f}_{\boldsymbol{m}}(\boldsymbol{m}) \text { with median } \\
\text { values of } \boldsymbol{m}_{\max } \text { and } \boldsymbol{b}\end{array}$ \\
\hline \multirow{2}{*}{ Zone 1} & $b$ & 0.63 & 0.11 & \\
& $m_{\max }$ & 7.02 & 0.16 & $0.6844 . e^{-0.63(m-3)}$ \\
\hline \multirow{2}{*}{ Zone 2} & $m_{\min }$ & 3 & - & \\
& $b$ & 1.16 & 0.07 & $1.167 . e^{-1.16(m-3)}$ \\
\hline \multirow{2}{*}{ Zone 3} & $m_{\max }$ & 7.37 & 0.12 & \\
& $m_{\min }$ & 3 & - & $1.173 . e^{-1.13(m-3)}$ \\
\hline
\end{tabular}

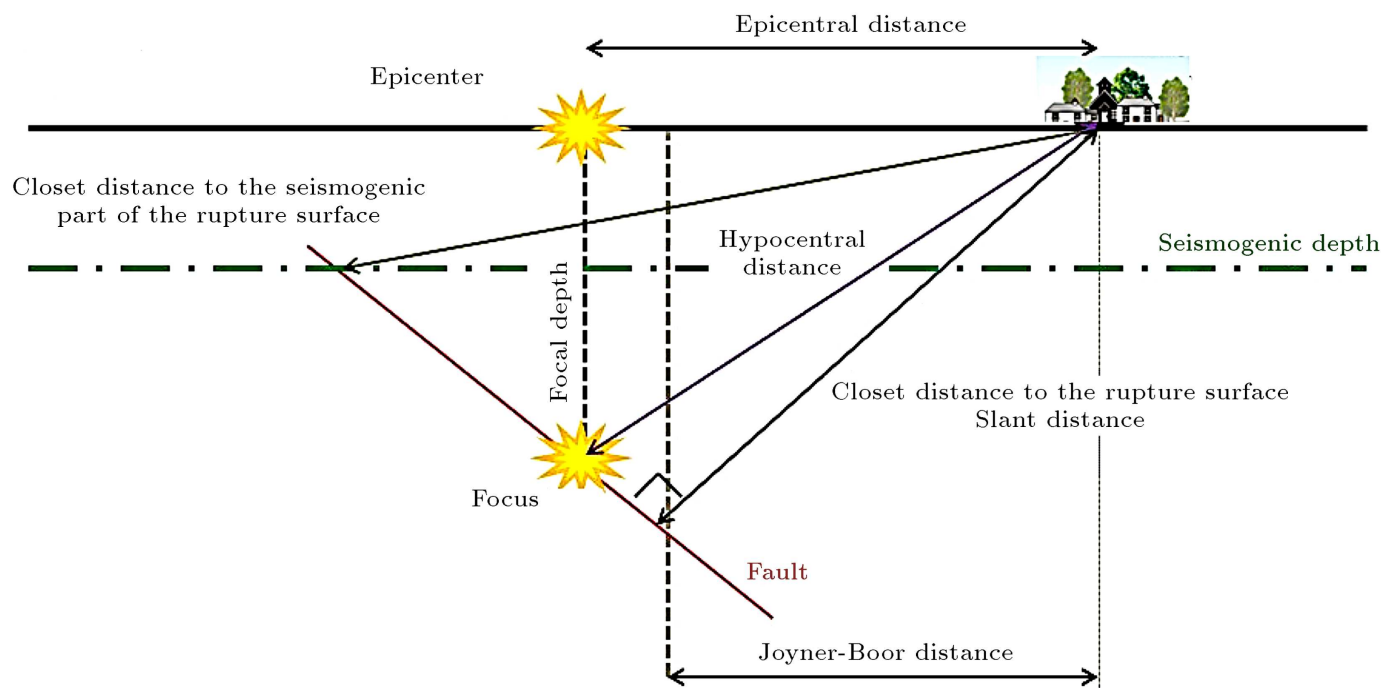

Figure 5. Graphical interpretation of the epicentral distance.

$$
\begin{aligned}
F_{R}(r) & =P\left(r_{\min }<R<r\right) \\
& = \begin{cases}0 & r<r_{\min } \\
\frac{\pi \cdot r^{2}-\pi r_{\min }^{2}}{\pi r_{\max }^{2}-\pi r_{\min }^{2}} & r_{\min } \leq r \leq r_{\max } \\
1 & r \geq r_{\max }\end{cases}
\end{aligned}
$$

According to Eq. (13), the probabilistic description of epicentral distance can be computed by:

$$
f_{R}(r)=\frac{\partial F_{R}(r)}{\partial r}= \begin{cases}\frac{2 \pi r}{\pi r_{\max }^{2}-\pi r_{\min }^{2}} & r_{\min } \leq r \leq r_{\max } \\ 0 & \text { Otherwise }\end{cases}
$$

Based on the above equation, epicentral probability function of the seismic zones is developed as follows:

$$
\begin{array}{ll}
f_{1}(r)=\frac{r}{1750} & 10 \leq r<60, \\
f_{2}(r)=\frac{r}{5400} & 60 \leq r<120, \\
f_{3}(r)=\frac{r}{4050} & 120 \leq r<150 .
\end{array}
$$

It is strongly emphasized that the above description is not only limited to circular area source, but also can be extended to line source or area source with a more general shape.

According to Eq. (9), to complete location model, focal depth also requires to be specified. Statistical analysis of focal depth values presented in published global earthquake catalogues shows that lognormal distribution is a suitable distribution function to define focal depth variability. However, focal depths published in seismic catalogues are inaccurate by up to $60 \mathrm{~km}$ for events in Iran and the surrounding regions [18]. Therefore, it is recommended that only focal depth values determined using accurate methods, such as local network recordings or teleseismic wave form inversion, be used instead of seismic catalogues values in a PSHA [18]. Therefore, based on a study conducted by Magge et al. [19], focal depth value, in this paper, is considered constant and set equal to $12 \mathrm{~km}$.

\subsection{Comparison of analysis options}

In this section, seismic hazard curve is derived using different reliability methods, and the results are 
then compared with those obtained from conventional method. The objective followed in this comparison is to find the appropriate reliability model for computing PSHA with respect to efficiency and accuracy criteria. To this end, for a given threshold, the exceedance probability is first calculated for each zone using different reliability methods. Next, the probability of exceedance is converted to the annual rate of exceedance by a simple modification. To this end, it is enough to multiply the probability by annual rate of occurrence of earthquake at the site. Finally, hazard curve is developed using direct summation of the annual rate of exceedance over all seismic zones. The reliability of the obtained results is investigated by its comparison with the results of conventional approach. To demonstrate differences between methods, vertical axes in Figure 6 are plotted in logarithmic space. According to Figure 6, FORM, especially at the tail of the curves, tends to overestimate the probability of failure compared with conventional and other reliability methods. This is quite reasonable and mainly due to the linearization of the limit-state function in the FORM. To examine the accuracy of the results better, $P G A$ is computed and compared with those obtained from conventional method in terms of percentage errors by applying Poisson occurrence model and reliability-based hazard curves, according to different occurrence probabilities in 50 years (Table 2). According to the results of Table 2, it is concluded that MCS is the most accurate method. Figure 6 graphically confirms this conclusion. However, it is too time-consuming to be used for practical purposes. To appreciate this fact, the number of simulations and $\mathrm{CPU}$ time required to compute the probability of exceeding a specific threshold value is shown in Table 3 for different reliability methods. According to the results of Table 3, the computational cost of MCS increases dramatically when the probability of rare event is sought. For example, to achieve $2 \%$ coefficient of variation of the probability results, MCS requires 750616 realizations for threshold equal to " $0.75 \mathrm{~g}$ " at the first seismic zone. The CPU time corresponding to these numbers of simulations is 5665 seconds, namely 1.57 hours. In return, the run time required to compute the probability of exceeding " $1.1 \mathrm{~g}$ " is equal to 32849.02 seconds which is more than 6 times of the run time required for the threshold equals " $0.75 \mathrm{~g}$ ". It is noteworthy that the aforementioned computational cost is relevant to only one threshold value. By considering computational efforts of different threshold values at all seismic zones, it is concluded that MCS is too time-consuming to be applied for practical purposes. In contrast, to compute the probability of exceeding " $0.75 \mathrm{~g}$ ", gradient-based methods, i.e. FORM and SORM (Breitung's improved formula), only 11 realizations are required which are equivalent to 0.102 and 0.149 seconds CPU time for FORM and
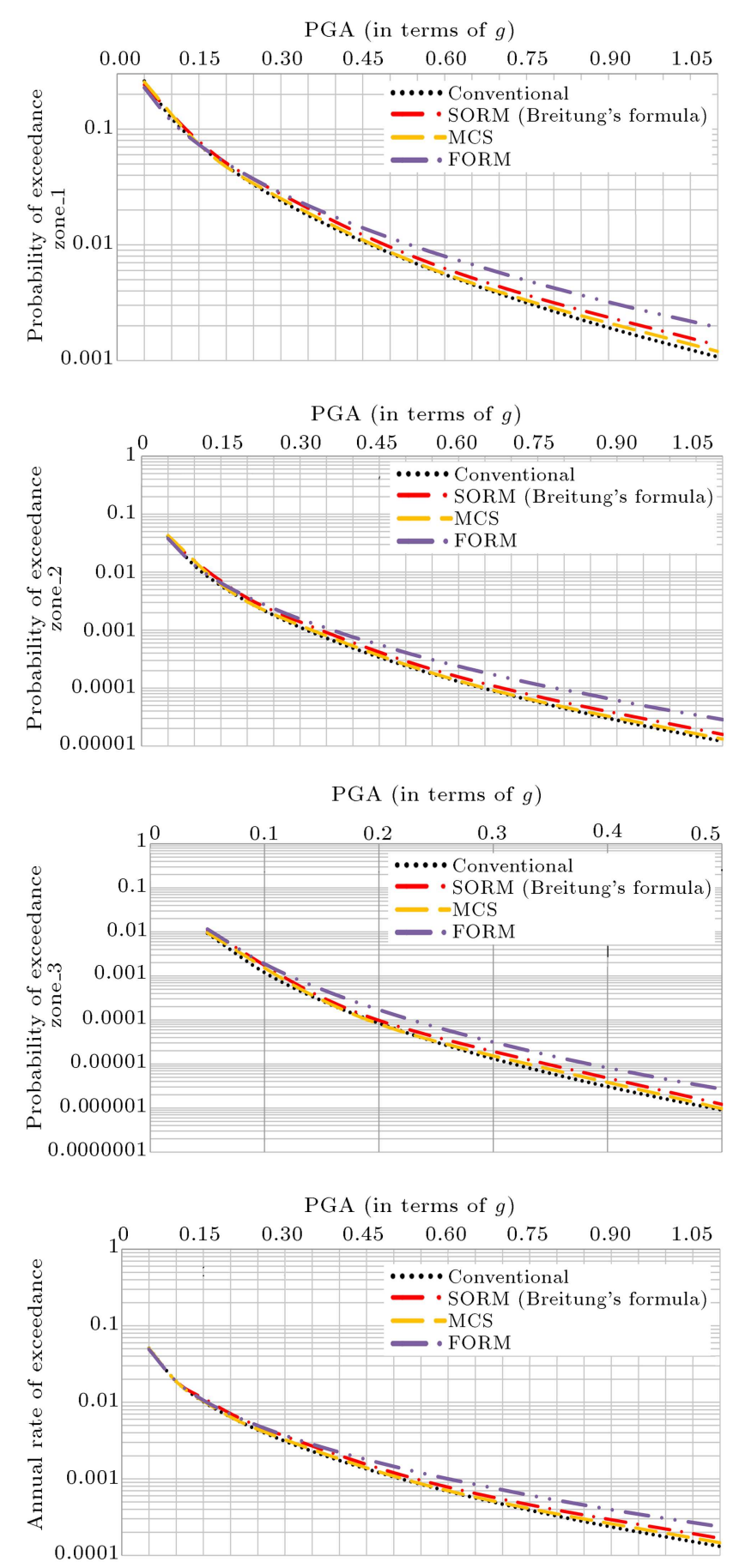

Figure 6. Comparison of the results obtained from different methods.

SORM, respectively. This brief comparison demonstrates how much faster the gradient-based methods are than MCS, which would be greatly interesting for practical purposes. However, according to Table 2, SORM provides more accurate results than FORM, especially at low probabilities. This is quite reasonable and mainly due to considering limit-state curvature when exceedance probability is sought. Therefore, the second-order reliability analysis based on Breitung's 
Table 2. $P G A$ according to different occurrence probabilities in 50 years.

\begin{tabular}{|c|c|c|c|}
\hline $\begin{array}{c}\text { Occurrence probability } \\
\text { during } 50 \text { year }\end{array}$ & $P G A_{\text {conventional method }}$ & $P G A_{\text {FORM }}$ & Error $^{a}$ \\
\hline 90 & 0.0572 & 0.0549 & $-4.021 \%$ \\
\hline 75 & 0.0859 & 0.0852 & $-0.815 \%$ \\
\hline 50 & 0.1285 & 0.1325 & $3.113 \%$ \\
\hline 25 & 0.2161 & 0.2305 & $6.664 \%$ \\
\hline 10 & 0.3668 & 0.4137 & $12.786 \%$ \\
\hline 2 & 0.7416 & 0.8967 & $20.914 \%$ \\
\hline $\begin{array}{c}\text { Occurrence probability } \\
\text { during } 50 \text { year }\end{array}$ & $P G A_{\text {conventional method }}$ & $P G A_{\text {Breitung's formula }}$ & Error $^{b}$ \\
\hline 90 & 0.0572 & 0.0569 & $-0.527 \%$ \\
\hline 75 & 0.0859 & 0.0858 & $-0.117 \%$ \\
\hline 50 & 0.1285 & 0.1291 & $0.465 \%$ \\
\hline 25 & 0.2161 & 0.2194 & $1.504 \%$ \\
\hline 10 & 0.3668 & 0.3735 & $1.794 \%$ \\
\hline 2 & 0.7416 & 0.781 & $5.045 \%$ \\
\hline $\begin{array}{c}\text { Occurrence probability } \\
\text { during } 50 \text { year }\end{array}$ & $P G A_{\text {conventional method }}$ & $P G A_{\mathrm{MCS}}$ & Error $^{c}$ \\
\hline 90 & 0.0572 & 0.05723 & $0.052 \%$ \\
\hline 75 & 0.0859 & 0.0861 & $0.233 \%$ \\
\hline 50 & 0.1285 & 0.1288 & $0.233 \%$ \\
\hline 25 & 0.2161 & 0.2177 & $0.740 \%$ \\
\hline 10 & 0.3668 & 0.3699 & $0.845 \%$ \\
\hline 2 & 0.7416 & 0.7516 & $1.33 \%$ \\
\hline \multicolumn{4}{|c|}{ Error $=\frac{P G A_{F O R M}-P G A_{\text {conventional method }}}{P G A_{\text {conventional method }}} \times 100 ;{ }^{b}$ Error $=\frac{P G A_{\text {Breitung's formula }}-P G A \text { conventional method }}{P G A_{\text {conventional method }}} \times 100 ;$} \\
\hline
\end{tabular}

Table 3. A comparison of computational efforts of the different reliability methods for computing probability of exceeding.

\begin{tabular}{cccccccc}
\hline \multirow{2}{*}{ Threshold } & \multicolumn{2}{c}{ MCS } & & \multicolumn{2}{c}{ FORM/SORM } \\
\cline { 3 - 4 } \cline { 7 - 8 } & & $\begin{array}{c}\text { Number of } \\
\text { evaluations }\end{array}$ & $\begin{array}{c}\text { CPU time } \\
\text { (Second) }\end{array}$ & & $\begin{array}{c}\text { Number of } \\
\text { evaluations }\end{array}$ & $\begin{array}{c}\text { CPU time } \\
\text { (Second) }\end{array}$ \\
\hline \multirow{2}{*}{ Zone 1 } & 0.25 & 71363 & 62.918 & & 5 & $0.077 / 0.1$ \\
& 0.5 & 286548 & 783.961 & & 8 & $0.086 / 0.116$ \\
& 0.75 & 750616 & 5664.16 & & 11 & $0.096 / 0.112$ \\
& 1.1 & 2080833 & 32849.02 & & 14 & $0.119 / 0.16$ \\
\hline
\end{tabular}

improved formula would be the authors' suggested reliability method for computing PSHA.

\section{Conclusion}

In this paper, an alternative approach based on the application of Reliability Methods is put forward to calculate Probabilistic Seismic Hazard Analysis (PSHA). The pioneering work (i.e., Eq. (1)) in PSHA is considered as a reference to exhibit efficiency and accuracy of the reliability methods. In both approaches, the results are peak ground acceleration hazard curves for the site at which a historical bridge in Iran is located. It is concluded that the subsequent use of the SORM (Breitung's improved formula) provides accurate results with respect to conventional method with a fraction of the computational efforts involved in MCS. That is, SORM can be adopted as an alternative approach to calculate PSHA because of good balance between accuracy and efficiency. The ability to explicitly account for epistemic uncertainties associated with the capability of effortlessly addressing correlation between random 
variables is another advantage, making the reliabilitybased approach powerful for practical purposes compared to conventional method. These advantages are particularly significant when attenuation relations with random model parameters are implemented in calculating PSHA.

\section{References}

1. Cornell, C.A. "Engineering seismic risk analysis", Bulletin of the Seismological Society of America, 58(5), pp. 1583-1606 (1968).

2. Wen, Y. "Probabilistic aspects of earthquake engineering", Earthquake Engineering from Engineering Seismology to Performance-Based Engineering, Ed. by Bozorgnia, Y. and V.V. Bertero, CRS Press LLC (2004).

3. Ditlevsen, O. and Madsen, H.O., Structural Reliability Methods, Wiley New York (1996).

4. Melchers, R.E., Structural Reliability Analysis and Prediction, John Wiley \& Sin Ltd (1999).

5. Haldar, A. and Mahadevan, S., Probability, Reliability, and Statistical Methods in Engineering Design, John Wiley \& Sons (2000).

6. Du, X. "ME/AE probabilistic engineering design", Class notes, Engineering Uncertainty Repository, http://web.mst.edu/ due/repository, 2016, unpublished.

7. Haukaas, T. "Unified reliability and design optimization for earthquake engineering", Probabilistic Engineering Mechanics, 23(4), pp. 471-481 (2008).

8. Du, X. "Unified uncertainty analysis by the first order reliability method", Journal of Mechanical Design, 130(9), pp. 091401-10 (2008).

9. Bohl, A. "Comparison of performance based engineering approaches", A Thesis Submitted in Partial Fulfillment of the Requirements for the Degree of Master of Applied Science, the University of British Columbia (2009).

10. Kiureghian, A.D. and Ditlevsen, O. "Aleatory or epistemic? Does it matter?", Structural Safety, 31(2), pp. 105-112 (2009).

11. Koduru, S. and Haukaas, T. "Probabilistic seismic loss assessment of a Vancouver high-rise building", Journal of Structural Engineering, 136(3), pp. 235-245 (2009).

12. Xiao, N.-C., Huang, H.-Z., Wang, Z. Pang, Y. and He, L. "Reliability sensitivity analysis for structural systems in interval probability form", Structural and Multidisciplinary Optimization, 44(5), pp. 691-705 (2011).

13. Mahsuli, M. and Haukaas, T. "Seismic risk analysis with reliability methods. Part II: Analysis", Structural Safety, 42, pp. 63-74 (2013).

14. Breitung, K. "Asymptotic approximations for multinormal integrals", Journal of Engineering Mechanics, 110(3), pp. 357-366 (1984).
15. Zare, M., Ghafory-Ashtiany, M. and Bard, P.Y. "Attenuation law for the strong motions in Iran", In Proceedings of the Third International Conference on Seismology and Earthquake Engineering, 1, pp. 345354 (1999).

16. Kijko, A. and Sellevoll, M.A. "Estimation of earthquake hazard parameters from incomplete data files. Part II. Incorporation of magnitude heterogeneity", Bulletin of the Seismological Society of America, 82(1), pp. 120-134 (1992).

17. Kijko, A. "Estimation of the maximum earthquake magnitude, m max", Pure and Applied Geophysics, 161(8), pp. 1655-1681 (2004).

18. Maggi, A., Priestley, K. and Jackson, J. "Focal depths of moderate and large size earthquakes in Iran", Journal of Seismology and Earthquake Engineering, 4(2-3), pp. 1-10 (2002).

19. Maggi, A., Jackson, J., Priestley, K. and Baker, C. "A re-assessment of focal depth distributions in southern Iran, the Tien Shan and northern India: Do earthquakes really occur in the continental mantle?", Geophysical Journal International, 143(3), pp. 629$661(2000)$.

\section{Biographies}

Mehdi Kia started his university studies in Babol Noshirvani University of Technology, Babol, Iran, in the field of Civil Engineering, in September 2004. Having finished his undergraduate study in 2008, he continued his studies as a graduate student in Sharif University of Technology in Structural Engineering. He successfully defended his thesis titled "Modeling Local Joint Flexibility using a new element added to the library of OpenSees software" to obtain his MS degree in December 2010. He was accepted as a PhD student in the University of Amir-Kabir, Iran, in 2012, where he worked on reliability analysis and its application on seismic risk evaluations. He is currently a Structural Engineer at Pars Gas Oil Company (P.O.G.C).

Mehdi Banazadeh obtained his BS (Civil Engineering) and MS (Earthquake Engineering) degrees from the University of Tehran with honor. He then pursued his $\mathrm{PhD}$ degree in the University of the Ryukyus in Japan and accomplished it in 2004. He has many years of experience in the next generation performancebased engineering and reliability analysis. He has been a faculty member in Amir-Kabir University of Technology in Tehran, Iran. He has authored and coauthored over 30 technical papers. His research area is mainly focused on seismic risk evaluation using reliability framework and nonlinear structural modeling and analysis. 